\title{
AN EXTENSION OF HELSON-EDWARDS THEOREM TO BANACH MODULES
}

\author{
SIN-EI TAKAHASI
Yamagata University
Department of Basic Technology
Faculty of Engineering
Yonezawa 992
JAPAN

(Received September 23, 1988)

ABSTRACT. An extension of the Helson-Edwards theorem for the group algebras to Banach modules over commutative Banach algebras is given. This extension can be viewed as a generalization of Liu-Rooij-Wang's result for Banach modules over the group algebras.

KEY WORDS AND PHRASES. Multiplier, Banach modules, bounded approximate identity, compact abelian group, completely regular.

1. INTRODUCTION.

Let $A$ be a commutative complex Banach algebra with a bounded approximate identity $\left\{u_{\lambda}\right\}$ of norm $B$ and denote by $\Phi_{A}$ the class of all nonzero homomorphisms of $A$ into the field of complex numbers. The space $\Phi_{A}$, with the Gelfand topology, is called the carrier space of $A$. Let $X$ be a Banach left A-module. A continuous module homomorphism of $A$ into $X$ is called a multiplier of $X$. We introduce a family $\left\{X_{\phi}: \phi_{Q} \Phi_{A}\right\}$ of Banach A-modules such that any multiplier $T$ of $X$ can be represented as a function $T$ on $\Phi_{A}$ with $\mathrm{T}(\phi) \varepsilon \mathrm{X}_{\phi}$ for each $\phi \varepsilon \Phi_{A}$. In this setting we give an extension of the He1sonEdwards theorem for the group algebras to Banach modules. We also observe that this extension can be viewed as a generalization of Liu-Roolj-Wang's result for Banach modules over the group algebras. We further consider a local property of multipliers when $A$ is completely regular.

2. REPRESENTATION THEOREM OF MULTIPLIERS.

For each $\phi \in \Phi_{A}$, let $M_{\phi}$ denote the maximal modular ideal of $A$ corresponding to $\phi$ and define

$$
x^{\phi}=\overline{s p}\left\{M_{\phi} X+\left(1-e_{\phi}\right) X\right\}
$$

where $\overline{\text { sp }}$ denotes the closed linear span and $e_{\phi}$ is an element of $A$ with $\phi\left(e_{\phi}\right)=1$. Note that $x^{\phi}$ does not depend on the choise of $e_{\phi}$. 
Throughout the remainder of this note we will assume

$$
\bigcap_{\phi \varepsilon_{A}} \overline{s p}\left(M_{\phi}\right)=\{0\} .
$$

In the case of $X=A$, the condition (2.1) is equivalent to the semisimplicity of $A$. The space $\overline{s p}(A X)$ is called the essential part of $X$ and is denoted by $X_{e} \cdot$ Since $A$ has a bounded approximate identity, it follows that $x_{e}=A x$ from the Cohen-Hewitt factorization theorem (see Doran-Wichman [1]). We also have

$$
x^{\phi} \quad x_{e}=\overline{s p}\left(M_{\phi} X\right)
$$

for all $\phi \in \Phi_{A^{*}}$ In fact, let $\phi \in \Phi_{A^{\prime}} x \in x^{\phi} x_{e}$ and $\varepsilon>0$. Since $x \varepsilon x^{\phi}$, there exist $a_{1}, \ldots, a_{n} M_{\phi}$ and $x_{1}, \ldots, x_{n}, y \quad x$ such that

$$
\left\|x-\sum_{i=1}^{n} a_{i} x_{i}-\left(1-e_{\phi}\right) y\right\|<\varepsilon / \beta \text {. }
$$

The'refore for each $\lambda$, we have

$$
\left\|u_{\lambda} x-\left(\sum_{i=1}^{n} u_{\lambda} a_{i} x_{i}+\left(u_{\lambda}-u_{\lambda} e_{\phi}\right) y\right)\right\|<\varepsilon .
$$

Letting $\varepsilon+0$, we obtain that $u_{\lambda} x \in \overline{\operatorname{sp}}\left(M_{\phi} x\right)$ for all $\lambda$. Since $x \in x_{e}, 1$ im $u_{\lambda} x=x$. Consequent1y, we have that $x \in \overline{\operatorname{sp}}\left(M_{\phi} X\right)$ and hence $x^{\phi} \cap x_{e} \subset \overline{s p}\left(M_{\phi} X\right)$. The reverse inclusion is immediate.

We denote by $M(A, X)$, or simply $M(X)$, the class of all multipliers of $X$. Then $M(X)$ also becomes a Banach A-module under the module multiplication defined by $(a T) b=a(T b)$. For each $x \varepsilon X$, the mapping $\tau_{x}$ of $A$ into $X$ defined by $\tau_{x}(a)=a x$ is a multiplier of $X$, so that $\tau$ becomes a module homomorphism of $X$ into $M(X)$. Also it can be easily observed that

$$
\mathrm{TA} \subset \mathrm{X}_{\mathrm{e}} \text { and } \mathrm{TM}_{\phi} \subset \overline{\mathrm{M}_{\phi} \mathrm{X}}
$$

for all $T \in M(X)$ and $\phi \in \Phi_{A}$, where the bar denotes the norm closure.

Now, for each $\phi \in \Phi_{A}$, let $x_{\phi}=x / x^{\phi}$ be the quotient of $x$ by $x^{\phi}$. So $x_{\phi}$ becomes a Banach A-module under the natural module structure and the quotient norm. For each $x \in X$, let $x(\phi)=x+X^{\phi}$ be the natural image of $x$ in $X^{\phi}$. A vector field on $\Phi_{A}$ is a function $\sigma$ defined on $\Phi_{A}$ with $\sigma(\phi) \in x_{\phi}$ for each $\phi \in \Phi_{A}$ of course, $\hat{x}(x \in x)$ is a vector field on $\Phi_{A}$. Denote by $\pi X_{\phi}$ the class of all vector fields on $\Phi_{A}$ and so $1 t$ becomes an A-module under the module multiplication defined by $(a \sigma)(\phi)=\hat{a}(\phi) \sigma(\phi)$, where a denotes the Ge1fand transform of a A. Define

$$
\pi^{b} x_{\phi}=\left\{\sigma \quad \pi x_{\phi}:|| \sigma\left|\|_{\infty}=\sup _{\phi \in \Phi_{A}}\right||\sigma(\phi)| \mid<+\infty\right\} .
$$

Then $\pi^{b} x_{\phi}$ beomes a Banach A-module under the norm \|\|$_{\infty}$ and $x=\{\hat{x}: x \in x\} \subset \pi^{b} x_{\phi}$. 
With the above notations, we have the following representation theorem of multipliers.

THEOREM 2.1. (i) If $\mathrm{T} M(\mathrm{X})$, then there exists a unique vector field $\hat{\mathrm{T}}$ on $\Phi_{\mathrm{A}}$ such that $\widehat{\mathrm{T} a}=\hat{\mathrm{T}}$ for all $\mathrm{a} \in \mathrm{A}$. (ii) The mapping $\mathrm{T}+\hat{\mathrm{T}}$ is a continuous module isomorphism of $M(X)$ into $\pi^{b} X_{\phi}$.

PROOF. Let $T \in M(X), a \in A$ and $\phi \in \Phi_{A} \cdot$ Since $e_{\phi} a_{\lambda}-\hat{a}(\phi) e_{\phi} u_{\lambda} \in M_{\phi}$ for all $\lambda$, it follows from (2.3) that $T\left(e_{\phi}{ }^{a u}{ }_{\lambda}\right)-\hat{a}(\phi) T\left(e_{\phi} u_{\lambda}\right) \in \overline{M_{\phi} X}$ for all $\lambda$. Hence, after taking the limit with respect to $\lambda$, we obtain $\mathrm{T}\left(\mathrm{e}_{\phi} \mathrm{a}\right)-\hat{\mathrm{a}}(\phi) \mathrm{Te} \boldsymbol{\phi}_{\phi} \in \overline{\mathrm{M}_{\phi} \mathrm{X}}$. Note also that $\mathrm{Ta} \in \mathrm{X}_{e}$ from (2.3). Then there exist $\mathrm{c} \varepsilon \mathrm{A}$ and $\mathrm{y} \varepsilon \mathrm{X}$ such that $\mathrm{Ta}=\mathrm{cy}$, so that

$$
\begin{aligned}
& \mathrm{T} a-T\left(e_{\phi} \mathrm{a}\right)=\mathrm{Ta}-\mathrm{e}_{\phi} \mathrm{Ta}=\left(\mathrm{c}-\mathrm{e}_{\phi} \mathrm{c}\right) \mathrm{y} \in \mathrm{M}_{\phi} \mathrm{X} \text {. We therefore have } \\
& \mathrm{Ta}-\hat{a}(\phi) \mathrm{Te} e_{\phi}=\left(\mathrm{Ta}-\mathrm{Te}_{\phi} \mathrm{a}\right)+\left(\mathrm{Te} \phi_{\phi} \mathrm{a}-\hat{\mathrm{a}}(\phi) \mathrm{Te}{ }_{\phi}\right) \in \mathrm{M}_{\phi} \mathrm{X}+\overline{M_{\phi} \mathrm{X}} \subset \overline{\operatorname{sp}}\left(M_{\phi} \mathrm{X}\right) \subset \mathrm{X}^{\phi} \text {. }
\end{aligned}
$$
Setting $\hat{T}(\phi)=\widehat{T e}_{\phi}(\phi)$, we obtain that $\widehat{\mathrm{Ta}}(\phi)=\hat{\mathrm{a}}(\phi) \hat{\mathrm{T}}(\phi)=(\mathrm{aT})(\phi)$. In other words, $\hat{\mathrm{T} a}$ $\hat{=} \hat{a T}$ for $a l l a \in \hat{A}$. If $\sigma \in \pi x_{\phi}$ such that $\hat{T a}=a \sigma$ for $a l 1 a \in A$, then $\hat{\mathrm{T}}(\phi)=\hat{\mathrm{Te}}_{\phi}(\phi)=\hat{e}_{\phi}(\phi) \sigma(\phi)=\sigma(\phi)$ for all $\phi \quad \Phi_{A}$, so that $\hat{T}=\sigma$. This proves (i). It is immediate from (i) that $T \rightarrow T$ is a continuous module homomorphism of $M(X)$ into $\pi^{\mathrm{b}} \mathrm{X}_{\phi^{*}} \wedge$ To show that this mapping is injective, let $\mathrm{T} \in M(X)$ with $\hat{T}=0$. Then $\widehat{\mathrm{TA}}=\hat{\mathrm{AT}}=\{0\}$ from (i), so TA $\bigcap_{\hat{\epsilon} \Phi_{A}} \mathrm{X}^{\phi}$. Also TAC $\mathrm{x}_{e}$ from (2.3). Therefore, by (2.2) and our assumption (2.1),

$$
\operatorname{TAC} \bigcap_{\phi \in \Phi_{A}} x_{e} \cap x^{\phi}=\bigcap_{\phi \in \Phi_{A}} \overrightarrow{s p}\left(M_{\phi} X\right)=\{0\}
$$

We thus obtain $T=0$, and (ii) is proved.

A Banach left A-module $X$ is said to be order-free if for every $x \in X$ with $x \neq 0$ there exists a $\in A$ with $a x \neq 0$.

COROLLARY 2.2. Let $x \in X$. If either $x \in x_{e}$ or $x$ is order-free, then $\hat{x}=0$ implies $\mathrm{x}=0$.

PROOF. Note first that

$$
\hat{a x}=a \hat{x}, a \in A, x \in X .
$$

In fact, for each $\phi \varepsilon \Phi_{A}$,

$$
a x-\hat{a}(\phi) x=\left(a-\hat{a}(\phi) e_{\phi}\right) x-\hat{a}(\phi)\left(1-e_{\phi}\right) x \in M_{\phi} x-\left(1-e_{\phi}\right) x \subset x^{\phi} \text {. }
$$

This implies (2.4). Now let $x \in X$ with $\hat{x}=0$. By the above theorem and (2.4), we have

$$
\hat{\tau}_{x}(\phi)=\hat{e}_{\phi}(\phi) \hat{\tau}_{x}(\phi)=\left(e_{\phi} \hat{\tau}_{x}\right)(\phi)=\hat{\tau}_{x} e_{\phi}(\phi)=\widehat{e}_{\phi} x(\phi)=\hat{e}_{\phi}(\phi) \hat{x}(\phi)=\hat{x}(\phi)
$$

for all $\phi \in \Phi_{A}$, so that $\hat{\tau}_{x}=\hat{x}_{\text {. Then }} \hat{\tau}_{x}=0$ and hence $A x=\{0\}$. Accordingly, if either $x \in X_{e}$ or $X$ is order-free, then $x=0$.

3. EXTENSION OF HELSON-EDWARDS THEOREM.

We give a characterization of multipliers of an order-free Banach A-module which is similar to [2, Theorem 1.2.4] and Liu, van Rooif, and Wang [3, Lemma 1.3]. 
COROLLARY 3.1. Let $X$ be order-free and $T$ a mapping of $A$ into $X$. Then the following conditions are equivalent.

(i) $\mathrm{T} \in \mathrm{M}(\mathrm{X})$.

(ii) $T$ is linear and continuous; $\mathrm{TM}_{\phi} \subset \mathrm{X}^{\phi}$ for every $\phi \subset \Phi_{A}$.

(iii) $\mathrm{T}(\mathrm{ab})=\mathrm{aTb}$ for $\mathrm{all} \mathrm{a}, \mathrm{b} E \mathrm{~A}$.

PROOF. (i) $\Rightarrow$ (ii) follows immediately from (2.3). (ii) $=\Rightarrow$ (iii). Let $a, b \in A$ and $\phi \dot{e} \Phi_{A} \cdot$ Since abu $-\grave{a}(\phi) b u_{\lambda} \leftarrow M_{\phi}$ for all $\lambda$, it follows from (ii) that $\mathrm{T}\left(\mathrm{abu}_{\lambda}\right)-\grave{a}(\phi) \mathrm{T}\left(\mathrm{bu}{ }_{\lambda}\right)-\mathrm{TM}_{\phi} \mathrm{X}^{\phi}$ for all $\lambda$. Hence, after taking the 1 imit with respect to $\lambda$, we obtain $\mathrm{T}(\mathrm{ab})-\hat{\mathrm{a}}(\phi) \mathrm{Tb} \in \mathrm{X}^{\phi}$. Then, by $(2.4), \widehat{\mathrm{T}(\mathrm{ab})}=\mathrm{a} \hat{\mathrm{Tb}}=\hat{\mathrm{aTb}}$, so that $\mathrm{T}(\mathrm{ab})=\mathrm{aTb}$ by Corollary 2.2 .

(iii) $\Rightarrow$ ( $i)$. To show that $T$ is 1 inear, let $a, b \leftarrow A$ and $\alpha, \beta$ scalars. Then

$$
\begin{aligned}
c \mathrm{~T}(\alpha a+\beta b) & =\mathrm{T}(\alpha \mathrm{ac}-\beta \mathrm{bc})=(\alpha \mathrm{a}+\beta \mathrm{b}) \mathrm{T} c=\alpha \mathrm{aTc}+\beta \mathrm{bTc} \\
& =\alpha c \mathrm{cT}+\beta c \mathrm{~T} b=c(\alpha \mathrm{Ta}+\beta \mathrm{T} b)
\end{aligned}
$$

for $a 11 c \in A$. Since $X$ is order-free, $T(\alpha a+\beta b)=\alpha T a+\beta T b$.

To show the continuity of $T$, let $1 \mathrm{im} a_{n}=a E A$ and $1 \mathrm{im} T a_{n}=x \in X$. Then

$$
\mathrm{bTa}=\mathrm{aTb}=1 \mathrm{im} \mathrm{a}_{\mathrm{n}} \mathrm{Tb}=1 \mathrm{im} \mathrm{bTa} \mathrm{a}_{\mathrm{n}}=\mathrm{bx}
$$

for $a 11 \mathrm{~b} \in \mathrm{A}$. So $\mathrm{Ta}=\mathrm{x}$ and hence $\mathrm{T}$ is continuous by the closed graph theorem.

Let $\widehat{M(A)}=\{\hat{T}: T \in M(X)\}$. The following result is an extension of the HelsonEdwards theorem for the group algebra of a locally compact Abelian group (see Rudin [4, Theorem 3.8.1]).

THEOREM 3.2. Let $\sigma \in \mathbb{I X}_{\phi^{\circ}}$ Then, $A \sigma \subset M(\bar{X})$ if and on $1 y$ if $\sigma \in \hat{M(X)}$.

PROOF. Note first that $\hat{\tau}_{x}=\hat{x}$ for all $x \in X$ as observed in the proof of Corollary 2.2. If $T \in M(X)$ with $\hat{T}=\sigma$, then, by Theorem 2.1 , a $\sigma=a \hat{T}=\hat{T} a=\hat{\tau}_{T a} C \dot{M}(X)$ for all a A.

Suppose conversely that $A \sigma \subset \overline{M(X)}$. Let a $\in A$. By the Cohen-Hewitt factorization theorem, $a$ can be written as $a=b c$ for some $b, c \in A$. Choose $s \leftarrow M(X)$ with $c \sigma=\hat{s}$. Then, $a \sigma=b c \sigma=b \hat{S}=\widehat{S b} \varepsilon \hat{x}_{e}$ from (2.3). Hence, by Corollary 2.2, there is a unique element of $x_{e}$, say $T a$, such that $a \sigma=\widehat{T a}$. If $a, b$ are arbitrary elements of $A$, then $\widehat{\mathrm{T}(\mathrm{ab})}=\mathrm{ab \sigma}=\mathrm{a}(\mathrm{b} \sigma)=\mathrm{aTb}=\hat{\mathrm{aTb}}$ by $(2.4)$. Since $\mathrm{TA} \mathrm{X}_{\mathrm{e}}, \mathrm{T}(\mathrm{ab})=\mathrm{aTb}$ by Corollary 3.1. Note that $X_{e}$ is an order-free Banach A-module. Then, by Corollary 3.1, $T \subset M\left(A, X_{e}\right) \subset M(A, X)=M(X)$. Consequent $1 y, \sigma=\hat{T} \in \hat{M}(X)$ and the theorem is proved.

We will observe that Theorem 3.2 can be viewed as a generalization of Liu-RooijWang 's result [3, Theorem 2.3$]$.

Let $\mathrm{G}$ be a compact Abelian group and X a Banach $L^{1}(\mathrm{G})$-module. Let $X_{\gamma}=\gamma X$ for each $\gamma \hat{G}$ the dual group of G. Also denote by $\pi X_{\gamma}$ the class of all mappings $\rho$ of $\hat{G}$ into X such that $\rho(\gamma) \in$ $X_{\gamma}$ for every $\gamma \in G$. Set $\emptyset_{\gamma}(f)=\hat{f}(\gamma) \quad\left(\gamma \in \hat{G}, f \in L^{1}(G)\right)$, where $\hat{f}$ is the Fourier transform of $f$. Note that for each $\gamma \in \hat{G}, X^{\emptyset_{\gamma}}=(1-\gamma) X$ and $X^{\emptyset_{\gamma}}$ is isometrically module-isomorphic to $X_{\gamma}$. Also since sp $\hat{G}=L^{1}(G)$, it follows that 


$$
\bigcap_{\gamma \in G} x^{\phi} \gamma=\{0\}
$$

and hence $X$ satisfies $(2.1)$. For each $x \in X$, denote by $\tilde{x}$ the restriction of $\tau_{x}$ to $\hat{G}$ and set $\tilde{x}=\{\tilde{x}: x \in X\}$.

COROLLARY (Liu-Rooij-Wang). $\rho \leqslant \pi X_{\gamma}$ can be extended to a multiplier of $X$ if and only if $\hat{f} \rho=\tilde{x}$ for every $f \in L^{l}(G)$.

PROOF. Clearly $\hat{\mathrm{E}}(\gamma) \gamma=\gamma * f\left(\gamma \in \hat{G}, \hat{f} \in L^{1}(G)\right)$. So if $\rho=T \mid \hat{G}$ for some $\mathrm{T} \in M(\mathrm{X})$, then $\hat{\mathrm{f}} \rho=\hat{\mathrm{T}} \in \tilde{\mathrm{X}}$ for every $\mathrm{f} \in \mathrm{L}^{l}(G)$. Suppose conversely that $\rho \in \mathbb{I X} \mathrm{X}_{\gamma}$ and $\hat{f}_{p}=\tilde{x}$ for every $f \in L^{1}(G)$. Then for each $f \in L^{l}(G)$, choose $x_{f} \in X$ with $\hat{f}_{\rho}=\tilde{x}_{f}$. set $\sigma\left(\phi_{\gamma}\right)=\widehat{\rho(\gamma)}\left(\phi_{\gamma}\right)$ for each $\gamma \in \hat{G}$. We then have

$$
\begin{aligned}
(f \sigma)\left(\phi_{\gamma}\right) & =\hat{f}(\gamma) \widehat{\rho(\gamma)}\left(\phi_{\gamma}\right)=\left(\tilde{x}_{f}(\gamma)\right)\left(\phi_{\gamma}\right) \\
& =\hat{\gamma} x_{f}\left(\phi_{\gamma}\right)=\hat{x}_{f}\left(\phi_{\gamma}\right)
\end{aligned}
$$

for all $\gamma^{\circ} G$ and $f \in L^{1}(G)$. Thus $f \sigma=\hat{x}_{f}\left(\hat{x}\right.$ for all $f \varepsilon L^{l}(G)$ and hence $\sigma=\hat{T}$ for some $T \in M(X)$ from Theorem 3.2. Therefore,

$$
\widehat{\rho(\gamma)}\left(\phi_{\gamma}\right)=\hat{T}\left(\phi_{\gamma}\right)=(\gamma \bar{T})\left(\phi_{\gamma}\right)=\hat{T} \gamma\left(\phi_{\gamma}\right) \text {, }
$$

so that $\rho(\gamma)-T_{\gamma} \varepsilon X^{\phi} \gamma=(1-\gamma) X$ for all $\gamma \in \hat{G}$. But $\rho(\gamma)$, Trt $\gamma X$ and so $\rho(\gamma)-\mathrm{T} \gamma \in \gamma \mathrm{X}$ for all $\gamma(\hat{G}$. Consequent $1 \mathrm{y}, \rho=\mathrm{T} \mid \hat{G}$.

4. LOCAL PROPERTIES OF MULTIPLIERS.

We will consider local properties of multipliers. To do this, we introduce the following notation which is exactly similar to one given in Rickart [5, 2.7.13].

DEFINITION. Let $\sigma \in{\pi X_{\phi}}_{\phi}$ and $\sum \subset \pi x_{\phi}$. Then $\sigma$ is said to belong to $\Sigma$ near a point $\phi E \Phi_{A}$ (or at infinity) provided there exists a neighborhood $V$ of $\phi$ (or infinity) and an element $\sigma^{\prime} \quad \sum$ such that $\sigma / V=\sigma^{\prime} \mid V$. If $\sigma$ belongs to near every point of $\Phi_{A}$ and at infinity, then $\sigma$ is said to belong locally to $\Sigma$.

The following result is similar to one given in [5, 2.7.16] and we refer to the proof of one.

THEOREM 4.1. Assume A to be completely regular and let $\Sigma$ be a submodule of $\operatorname{IX}_{\phi}$. If $\sigma \varepsilon \pi X_{\phi}$ belongs locally to $\Sigma$, then $\sigma \in \Sigma$.

PROOF. Since $\sigma$ belongs to $\Sigma$ at infinity, there exists an open set $U_{0}$ of $\Phi_{A}$ with compact complement $K$ and $\sigma_{0} f . \Sigma$ with $\sigma_{0}\left|U_{0}=\sigma\right| U_{0}$. Also since $\sigma$ belongs to $\Sigma$ near every point of $K$, there exists a finite open covering $\left\{U_{1}, \ldots, U_{n}\right\}$ of $K$ and a finite subset $\left\{\sigma_{1}, \ldots, \sigma_{n}\right\}$ of $\Sigma$ with $\sigma_{i}\left|U_{1}=\sigma\right| U_{1}(1=1, \ldots, n)$. Note that A admits a partion of the identity (cf. [5, Theorem 2.7.12]). Then there exists $e_{1}, \ldots, e_{n} A$ such that $e=e_{1}+\ldots+e_{n}$ is an identity for $A$ modulo $\operatorname{ker} x$ and $e_{i} \in \operatorname{ker}\left(\Phi_{A}-U_{i}\right)$ $(1=1, \ldots, n)$, where ker $K$ denotes the kernel of $k$. Set 


$$
\sigma^{\prime}=(1-e) \sigma_{0}+e_{1} \sigma_{1}+\ldots+e_{n} \sigma_{n} .
$$

Then $\sigma^{\prime}$ is obviously in $\Sigma$. We further assert $\sigma^{\prime}=\sigma$. In fact, if $\phi \in U_{0}$, then we have

$$
\begin{aligned}
\sigma^{\prime}(\phi) & =(1-\hat{e}(\phi)) \sigma(\phi)+\sum_{\phi \in U_{i}} \grave{e}_{i}(\phi) \sigma(\phi) \\
& =\left(1-\grave{e}(\phi)+\sum_{\phi \in U_{i}} \hat{e}_{i}(\phi)\right) \sigma(\phi) \\
& =\sigma(\phi) .
\end{aligned}
$$

If $\phi=k$, then $\hat{e}(\phi)=1$ and $\left\{i: 1<i<n, \phi \in U_{i}\right\} \neq \emptyset$, so that

$$
\begin{aligned}
\sigma^{\prime}(\phi) & =\sum_{\phi C U_{i}} \grave{e}_{i}(\phi) \sigma_{i}(\phi)=\sum_{\phi \in U_{i}} \hat{e}_{i}(\phi) \sigma(\phi) \\
& =\hat{e}(\phi) \sigma(\phi)=\sigma(\phi) .
\end{aligned}
$$

Consequent1y, $\sigma^{\prime}=\sigma$ and the theorem is proved.

Because $M(X)$ is a submodule of $\pi X_{\phi}$, we obtain the following local property of multipliers from the preceding theorem.

COROLLARY 4.2. Assume A to be completely regular. If $\sigma \in \Pi X_{\phi}$ belongs locally to $M(X)$, then $\sigma \in M(X)$.

Let $A$ contain local identities (cf. $[5,3.6 .11]$ ) and $T \in M(X)$. The closure of $\left\{\phi \in \Phi_{A}: \hat{T}(\phi) \neq 0\right\}$ is called the support of $T$ and is denoted by supp $T$. If supp $T$ is compact, then there exists a unique $x \in x_{e}$ with $T=\tau_{x}$. In fact, by [5, Theorem 3.6.13], A has an identity for A modulo $\operatorname{ker}(\operatorname{supp} \hat{T})$, say e. Set $x=$ Te. So the desired result follows from Theorem 2.1 and Corollary 2.2.

Similarly, we obtain that for each compact set $K$ of $\Phi_{A}$, there exists $x \in X_{e}$ with $\hat{T}|K=\hat{x}| K$.

\section{REFERENCES}

1. DORAN, R.S. and WICHMANN, J., Approximate Identities and Factorization in Banach Modules, Lecture Notes in Math. 768, Springer-Ver1ag, New York-Heidelberg, 1979.

2. LARSEN, R., An Introduction to the Theorey of Multipliers, Springer-Verlag, New York-Heide1berg, 1971 .

3. LIU, T.S., VAN ROOIJ, A.C.M. and WANG, J.K., A Generalized Fourier Transformation for $\mathrm{L}_{1}(\mathrm{G})$-modules, J. Austra1. Math. Soc. (Series A) 36 (1984), 365-377.

4. RUDIN, W., Fourier Analysis on Groups, New York, N.Y.: Interscience Publishers, Inc., 1962.

5. RICKART, C.E., General Theorey of Banach Algebras, Van Nostrand, Princeton, N.J.. 1960 . 


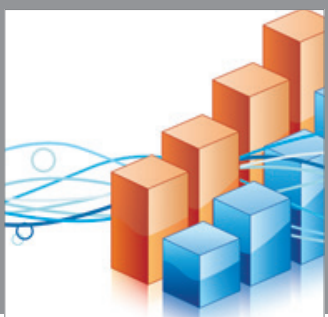

Advances in

Operations Research

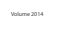

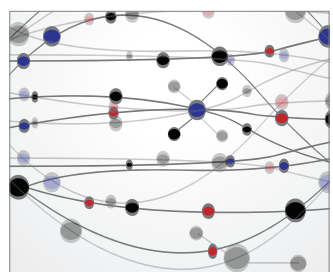

\section{The Scientific} World Journal
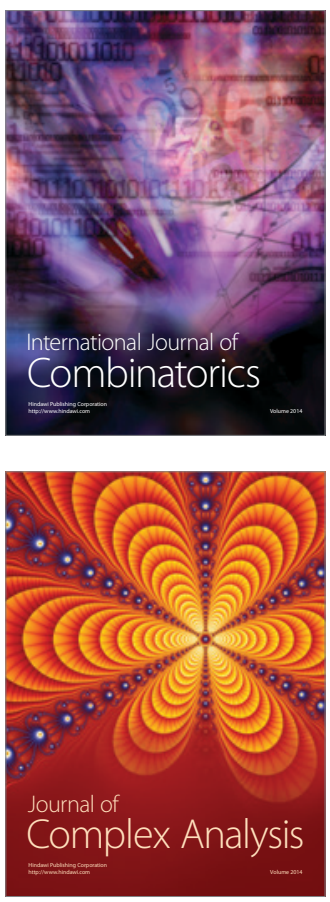

International Journal of

Mathematics and

Mathematical

Sciences
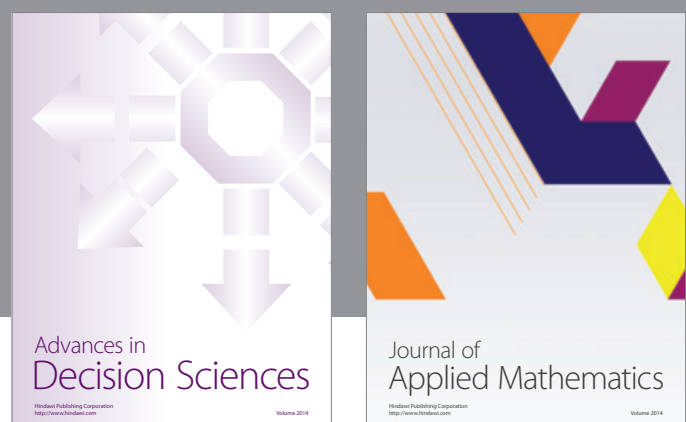

Journal of

Applied Mathematics
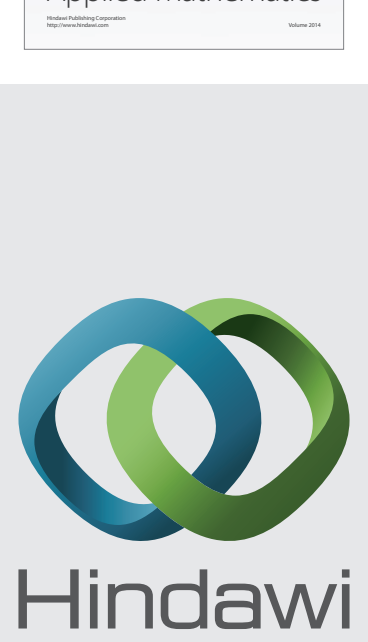

Submit your manuscripts at http://www.hindawi.com
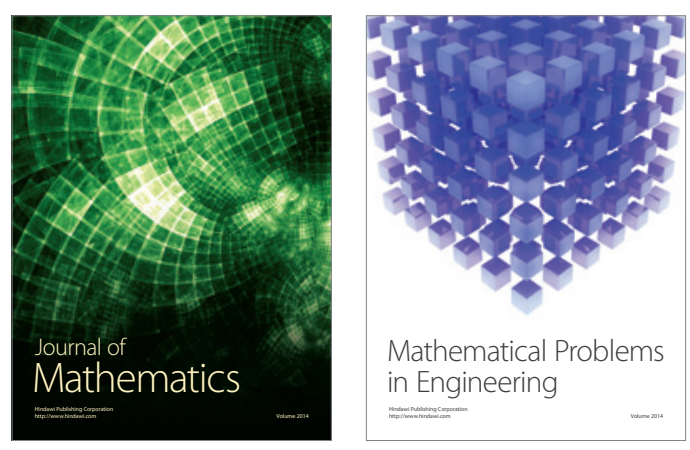

Mathematical Problems in Engineering


Journal of

Function Spaces
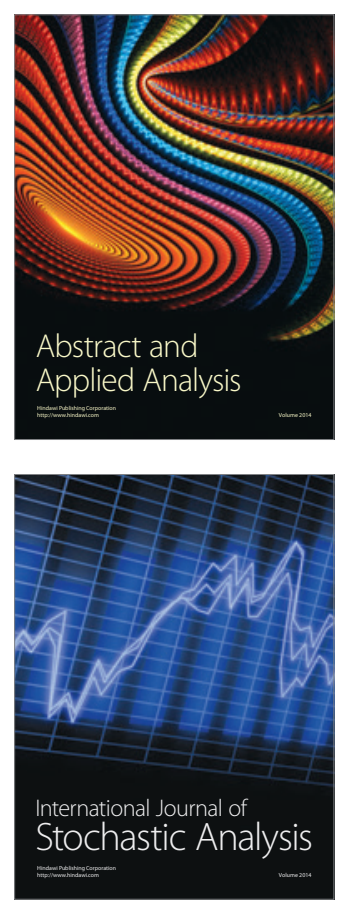

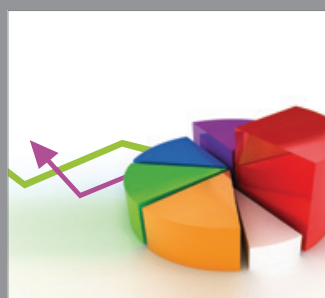

ournal of

Probability and Statistics

Promensencen
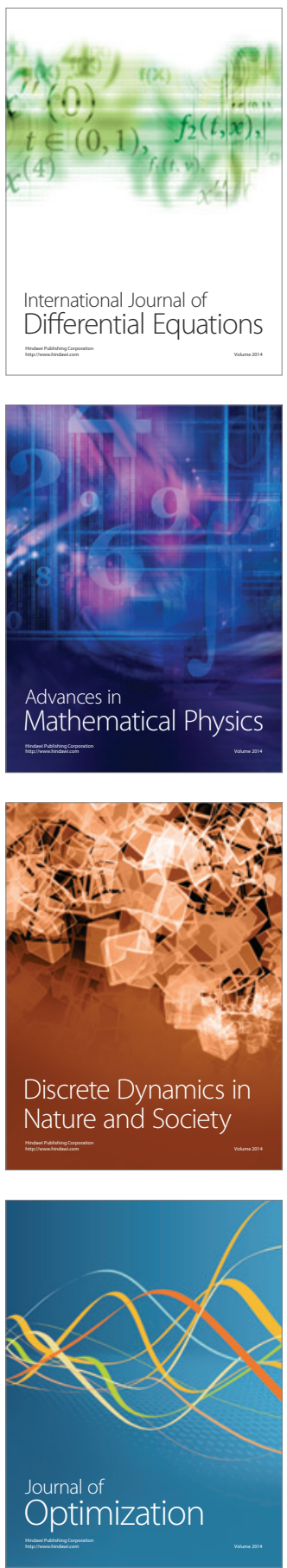